\title{
Addressing impacts of heterotopic ossificans through seating and positioning for individuals with spinal cord injury: A case study
}

\author{
Robyn Buckner* \\ Rancho Los Amigos National Rehabilitation Center, USA
}

\begin{abstract}
Introduction: Mature heterotopic ossificans of the hip joint can significantly impact sitting posture, comfort, sitting stability, pressure injury development, and functional activity for wheelchair users. When surgical interventions are deferred, modifications to the wheelchair seating system may serve as an alternative option to optimize positioning.

Background: In its mature phase, heterotopic ossificans, bony formation within soft tissue, create ankylosing at the joint. During this phase, surgical intervention is the only option for restoring passive range of motion at the joint. Non-surgical candidates are left with minimal alternative options and rely heavily on clinicians with knowledge in seating and positioning to address these impairments.

Presentation of Findings: Patient with history of T4 complete SCI presented with left hip ROM of 10-30 degrees secondary to HO recurrence. To accommodate the patient's limited left hip ROM, a 4" high x 16" wide x 18 " long poly float foam cushion was fabricated with a left femur trough to level the pelvis. A cut out for the pelvis to offload bilateral ischial tuberosities and the Sacro coccyx region was also created in the cushion to decrease risk of skin breakdown. These modifications improved the patient's balance for sitting stability and use of bilateral upper extremities for performing bimanual tasks.
\end{abstract}

Discussion: Instead of reclining the wheelchair backrest, customized contours of a cushion can accommodate postural deformities; and thus, provide upright posturing and stability as a compensatory strategy for individuals who lack trunk control.

Conclusion: Through a comprehensive evaluation, modifications of an individual's personal seating system to accommodate fixed and/or semi-fixed postural deformities caused by mature HO improved stability, balance, and upright posture.

\section{Introduction}

For individuals who use a wheelchair for mobility, like those with spinal cord injury, optimal seating and positioning play an important part in maximizing comfort, stability, and functional activity $[1,2]$. Studies show, $20-30 \%$ of those with spinal cord injury will develop heterotopic ossificans (HO), bony formation within soft tissue, resulting in a range of motion reduction [3]. 70-97\% will occur at the hip joint [3]; thus, resulting in postural malalignment in the sitting position and pressure ulcer development [4]. A common presentation is a contralateral pelvic obliquity, posterior pelvic tilt, decreased lumbar lordosis, ipsilateral side bend on the contralateral side, and increased thoracic kyphosis. This posturing results in instability, increased risk of pressure injuries [3-6], and decreased participation in activities for wheelchair users $[2,4]$. When surgical interventions are non-optimal or postponed for a future date, a conservative approach of modifying an individual's wheelchair seating system may serve as an alternative option to optimize positioning and prevent the development or progression of pressure injury development.

\section{Background}

For wheelchair users, customized seating to provide upright trunk positioning are important concepts for optimizing an individual's independence, posture, comfort, and participation in daily life roles [2]. A comprehensive seating and mobility evaluation including a thorough subjective interview, an objective seated and supine assessment, and an understanding of the individual's personal factors, skin integrity, and functional level should be considered when selecting wheelchair parts and support surfaces for patients [2]. Understanding of the importance of these assessments in addition to the implications of findings are even more appreciated when working with individuals who present with significant hip, trunk, and pelvic asymmetries which are com promising their overall level of function and quality of life.

Heterotopic ossificans, bony formations developed and deposited within soft tissue of primarily large joints such as the hip, knee, shoulder, and elbow, is a common secondary complication seen most in individuals with severe peripheral or central nervous system injuries [7]. According to available research, the incidence of heterotopic ossificans in persons with history of traumatic spinal cord injury ranges from $15-30 \%$ [8] with higher occurrence individuals with spasticity, thoracic trauma, complete lesion, pneumonia, tracheostomy, nicotine

*Correspondence to: Robyn Buckner, Rancho Los Amigos National Rehabilitation Center, California, USA, E-mail: srubayi@dhs.lacounty.gov

Key words: heterotopic ossificans, wheelchair seating and positioning, spinal cord injury, paraplegia, tetraplegia

Received: May 21, 2018; Accepted: June 09, 2018; Published: June 13, 2018 
use, and urinary tract infection [9,10]. In the acute phase, HO presents with pain, inflammation, swelling, redness, and warmth; paralleling many of the signs and symptoms of a DVT, osteomyelitis, or septic joint $[3,7]$. For this reason, it is most often not diagnosed until its maturity phase when ankylosing of the joint has occurred [7].

While HO detection using three-phase technetium bone scan or X-ray imaging can allow for early intervention in preventing or decreasing the rate of ankylosis $[3,4]$, once $\mathrm{HO}$ has reached the stage of maturity, surgical intervention, using techniques such as the Girdlestone procedure [4], is the only effective treatment to regain passive mobility within the joint $[4,7]$. This procedure prevents sequelae of secondary postural deformities which can lead to compromise in balance, skin, and function. However, it comes with risk of surgical hemorrhage, fracture, and recurrence [7]. In a study conducted by Law-ye et al, in which 101 consecutive patients with hip $\mathrm{HO}$ were screened by a surgeon using CT analysis, $6 \%$ of the 86 surgical excisions performed on a total of 80 patients were found to result in HO recurrence. Through statistical analysis within the same study, osteomas touching or disrupting the joint capsule, joint space narrowing, and demineralization where three characteristics found in the incidences of recurrence [7].

Despite the low recurrence rate for those who elected surgery, non-surgical candidates are unfortunately left with lifelong secondary deformities such as scoliosis and pelvic obliquities with tertiary complications including skin compromise, decreased unsupported balance, and decreased functional mobility. For this reason, the case study explores a conservative approach which was used to help restore comfort, position, balance, and function to an individual who is a fulltime power wheelchair user.

\section{Case presentation and PT evaluation}

\section{Case presentation}

This patient was a 42-year-old male with two-year history of T4 complete spinal cord injury, traumatic brain injury, and right upper extremity brachial plexus injury. The patient underwent a previous partial osteotomy of the left femur and heterotopic bone bordering the left femur due to presence of mature HO. Recent pelvic $\mathrm{x}$-ray revealed $\mathrm{HO}$ reaccumulating along the left remnant femoral head, greater trochanter, and proximal diaphysis of the femur. Despite the patient's preference toward surgery to excise the $\mathrm{HO}$ and regain ROM in his left hip for improved balance and positioning within his power wheelchair, he was not a candidate for surgery. In order to address the patient's goals of improved balance and positioning while maintaining his ability to assist with lateral transfers, a referral was made to physical therapy.

\section{Physical therapy evaluation}

A clinical risk assessment incorporating the ICF model as well as a physical assessment in both supine and sitting were conducted by the physical therapist to provide foundational information regarding the patient's preferences and impairments [2]. In sitting, the patient was found to present with posterior pelvic tilt, right pelvic obliquity, right thoracolumbar $\mathrm{C}$ curve, excessive upper thoracic kyphosis, right shoulder elevation with scapular protraction and upward rotation, forward head with left side bend and right rotation. Despite having bilateral trunk supports, the patient relied heavily on his right forearm resting on his armrest to support himself in sitting and was unable to simultaneously elevate his arms without losing balance to the right. In the supine position, the patient's passive range of motion of the left hip and knee were found to be $10-30$ degrees and 0-65 degrees respectively and within functional limits for sitting on the right. The patient also had semi-flexible pelvis (lacking 10 degrees to neutral) (Figures 1-4).

\section{Management}

Range of motion and manual therapy techniques are not options in mature H.O. due to the nature of the mature HO. Instead, the patient was educated on his postural deformities and how modifications made through his power wheelchair and sitting support surface could accommodate his postural deformities $[1,2]$, which in turn could improve his balance and stability. While he was agreeable to modifying his wheelchair and cushion, the patient did not want the modifications to compromise his ability to assist with lateral transfers using a transfer board.

An off-the-shelf fluid cushion with a contoured, stable base would not allow accommodation of his left fixed hip in extension. For this same reason, an off-the-shelf hybrid cushion (i.e. foam and air or foam and fluid insert) was determined inappropriate. While the offthe-shelf air cell cushion would provide accommodation at the hip and pelvis through immersion of his pelvis and fixed femur extension, the air created an unstable surface which increased the level of assistance required to perform a lateral transfer. Likewise, a custom cushion would allow accommodation by recessing the surfaces beneath the left hip and

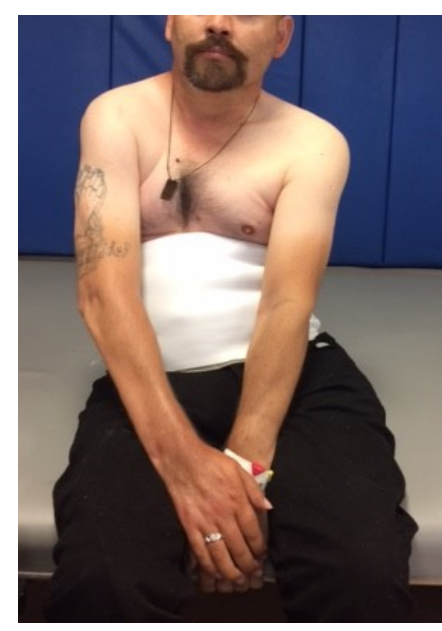

Figure 1. Anterior

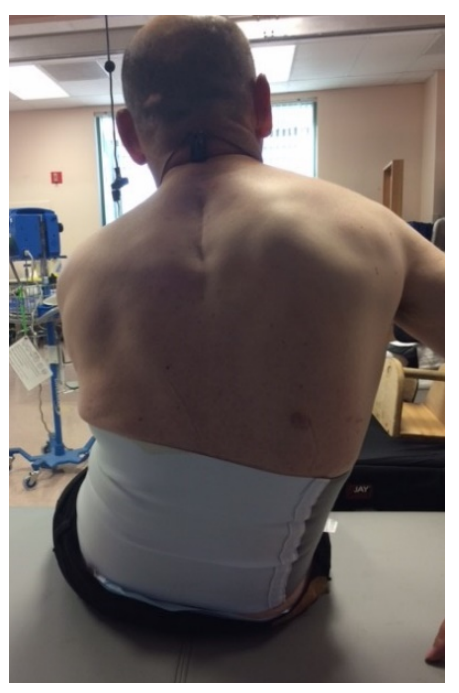

Figure 2. Posterior 


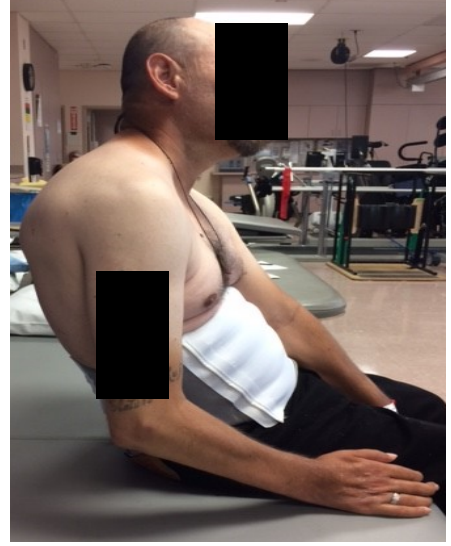

Figure 3. Side View

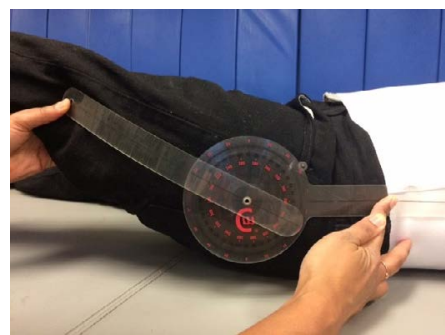

Figure 4. Appreciation of patient's left hip flexion.

pelvis; however, the many deep contours of a custom molded cushion would also diminish the patient's ability to perform his marginal transfers with a transfer board. As an alternative, a 4" high x 16" wide $\mathrm{x} 18$ " long, flat pressure relieving cushion was constructed out of 3 " high 5 \# foam and a 1 " high 4 \# foam laminated on top to meet both the anatomical and functional needs of the patient.

First a left femur trough was cut into the foam to accommodate the patient's available hip angle. Once sitting on the cushion with the trough cut out, the left footrest was lowered to further accommodate the change in the position of the extended lower extremity. The bony prominences along the patient's pelvis was palpated with corresponding areas of the cushion plucked and then recessed by making a 7" wide $\mathrm{x}$ 5" long x 3" deep cut out to completely off load the ischial tuberosities and Sacro coccyx region secondary to absent sensation and motor paralysis below the T4 level; decreasing the risk of skin breakdown[11]. Following these modifications, the patient was noted to present with a more leveled pelvis, upright trunk posture with his shoulders over his hips demonstrating the ability to perform bimanual tasks as well as outstretch his arms without loss of balance [12]. He maintained ability to perform lateral transfers with same level of assistance because the support surface was flat and firm. The patient reported increased stability [12] within his power wheelchair and overall satisfaction with modifications made to his seating system. During a separate visit, a similar configuration was made to his previous hybrid cushion made of a contoured foam base and fluid insert; however, the patient verbalized preference toward continued use of the foam cut out cushion due to its perceived comfort and stability. Patient continued to be monitored throughout his therapy visits without additional modifications needed to the cushion (Figures 5-7).

\section{Discussion}

While modifying the design of an individual's seating system does not increase the available range of the hip, the customized contours of the cushion can accommodate postural deformities. By making a trough and cutout for the ischial tuberosities, additional stability was provided to the wheelchair user by leveling his pelvis and lowering his

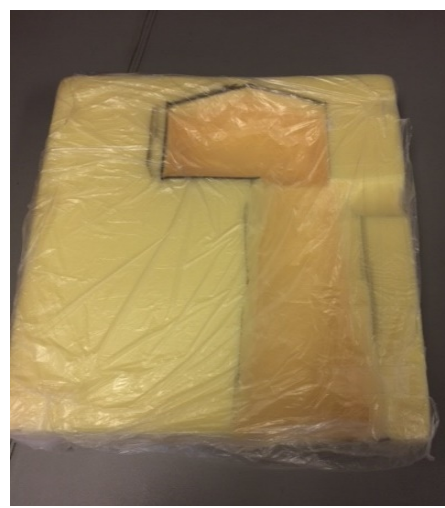

Figure 5. Foam polyfloat cushion with left trough and cut out for offloading ischial tuberosities and sacroco

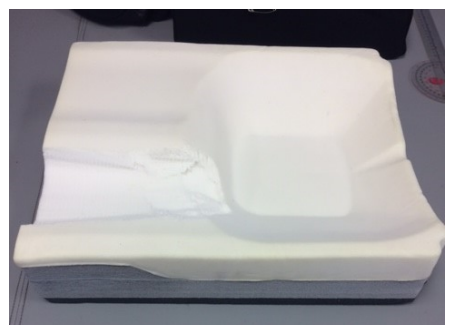

Figure 6. Modified hybrid cushion with left thigh trough

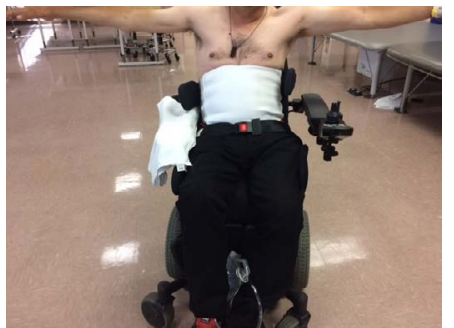

Figure 7a. Balance following new fabricated, foam cushion with outstretched arms

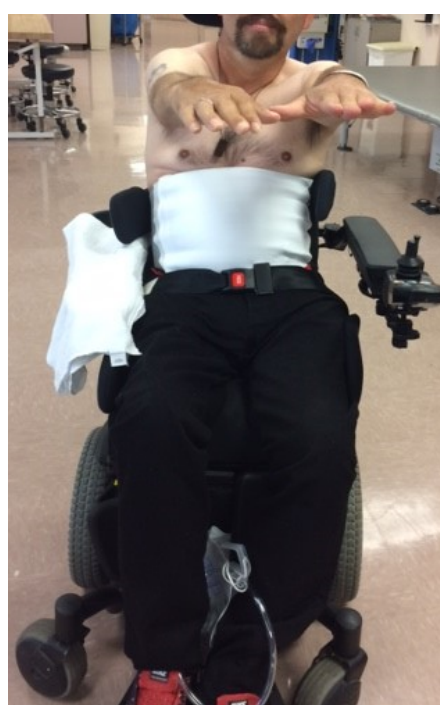

Figure 7b: Balance following new fabricated, foam cushion with for arms forward 
center of gravity [12]. This stability provides a compensatory strategy for individuals who lack trunk control; allowing the ability to maintain an upright posture which then allows balance and upper extremity function. This translated into improved movement including functional reach, time up in wheelchair, and comfort. Whereas, reclining the trunk to accommodate to the hip extension deformities would decrease his upright positioning and allow the pelvis to slide forward, decreasing stability and upper extremity function.

In less severe cases of individuals with $\mathrm{HO}$ of the hip, a combination of other methods can be used to provide similar accommodations as the one shown in this case study. This includes, but not limited to, reclining the back angle, changing the cushion medium to air, or altering the individual's personal cushion. However, the main concepts of fully flexing the hip and maintaining this available range to prevent the pelvis from sliding forward and allowing the trunk to maintain upright by providing a trough for the femur to compensate for the unavailable hip range cannot be lost. In the end, a clear understanding of the individual's impairments and how accommodation of one impairment effects another can help guide the clinician toward the best approach.

\section{Conclusion}

This case study provides one example of how modifications to a wheelchair seating system can accommodate deformities at the hip to improve trunk posture. Knowledge of the patient's preferences, various wheelchair cushion types and wheelchair adjustability as well as whether or not the patient's contractures and deformities are reducible vs fixed can lead a clinician toward a good, better, and best option to meet her or his patient's needs.

\section{References}

1. Ferguson-Pell MW (1990) Seat cushion selection. J Rehabil Res Dev Clin Suppl 2: 49-73. [Crossref]
2. Minkel JL (2000) Seating and Mobility Considerations for People with Spinal Cord Injury. Phys Ther: 80: 701-709. [Crossref]

3. van Kuijk AA, Geurts AC, van Kuppevelt HJ (2002) Neurogenic ossification in spinal cord injury. Spinal Cord 40: 313-326. [Crossref]

4. Rubayi S, Gabbay J, Kruger E, and Ruhge K (2016) The Modified Girdlestone Procedure with Muscle Flap for Management of Pressure Ulcers and Heterotopic Ossification of the Hip Region in Spinal Injury Patients: A 15-Year Review with Longterm Follow-up. Ann Plast Surg 77: 645-652. [Crossref]

5. Shabshin N, Ougortsin V, Zoizner G, Gefen A (2010) Evaluation of the effect of trunk tilt on compressive soft tissue deformations under the ischial tuberosities using weightbearing MRI. Clin Biomech 25: 402-408.

6. Gefen A (2014) Tissue changes in patients following spinal cord injury and implications for wheelchair cushions and tissue loading: a literature review. Ostomy Wound Manage 60: 34-45. [Crossref]

7. Law-ye Bruno, Hangard C, Felter A, Dominique Safa, Philippe Denormandie, et al. (2016) Pre-surgical CT-assessment of neurogenic myositis ossificans of the hip and risk factors of recurrence: a series of 101 consecutive patients. BMC Musculoskelet Disord 17: 433. [Crossref]

8. Cassar-Pullicino V, McClelland M, Badwan D, Pringle R, EI Masry W (1993) Sonographic diagnosis of heterotopic bone formation in spinal injury patients. International Medical Society of Paraplegia 31: 40-50.

9. Citak M, Suero EM, Backhaus M, Aach M, Godry H, et al. (2012) Risk factors for heterotopic ossification in patients with spinal cord injury: a case-control study of 264 patients. Spine (Phila Pa 1976) 37: 1953-1957. [Crossref]

10. Suero EM, Citak M (2018) Clinical Prediction Rule for Heterotopic Ossification of the Hip in Patients with Spinal Cord Injury. Spine (Phila Pa 1976). [Crossref]

11. Crane B, Wininger M, Call E (2016) Orthotic-Style Off-Loading Wheelchair Sea Cushion Reduces Interface Pressure Under Ischial Tuberosities and Sacrococcygeal Regions. Arch Phys Med Rehabil 97: 1872-1879. [Crossref]

12. Aissaoui R, Boucher C, Bourbonnais D, Lacoste M, Dansereau J (2001) Effect of seat cushion on dynamic stability in sitting during a reaching task in wheelchair users with paraplegia. Arch Phys Med Rehabil 82: 274-281. [Crossref]

Copyright: (C2018 Buckner R. This is an open-access article distributed under the terms of the Creative Commons Attribution License, which permits unrestricted use, distribution, and reproduction in any medium, provided the original author and source are credited. 